\title{
Traumatic Macular Hole Repair Following Surgery for Subretinal Hemorrhage
}

\author{
Zeynep Alkin, Abdullah Ozkaya, Asli Kirmaci, Gurkan Erdogan, Irfan Perente \\ Beyoglu Eye Training and Research Hospital, Istanbul, Turkey
}

\begin{abstract}
Macular holes can develop as result of intraocular inflammatory disease, trauma, or vitreous detachment, among other reasons. Presently described is case of full-thickness macular hole (FTMH) repair after successful surgery for traumatic submacular hemorrhage. FTMH may have developed due to injection of small air bubble into the subretinal space during surgery to remove subretinal hemorrhage. FTMH was successfully treated with pars plana vitrectomy and internal limiting membrane (ILM) removal using gas tamponade, as in cases of traumatic/idiopathic macular hole.
\end{abstract}

Keywords: Pars plana vitrectomy, subretinal hemorrhage, traumatic macular hole.

\section{Introduction}

Full-thickness macular holes (FTMH) can develop as result of intraocular inflammatory disease, trauma, or vitreous detachment, among other causes. Traumatic macular hole (TMH) may occur due to vitreomacular traction, subfoveal hemorrhage, or physical response to injury $(1,2)$.

Herein, we report an unusual case of FTMH and its repair after successful surgery for subretinal hemorrhage.

\section{Case Report}

An otherwise healthy 16-year-old boy presented with reduced central vision in his right eye occurring immediately after blunt trauma. Assessment of best corrected visual acuity (BCVA) showed $20 / 100$ in the right eye compared with $20 / 20$ in the left. Intraocular pressure was $12 \mathrm{mmHg}$ in both eyes. Anterior segment examination of both eyes was unremarkable. Fundus examination of the right eye showed subretinal hemorrhage at the posterior pole extending to the optic disc and slight vitreous hemorrhage.
No retinal tear or retinal detachment was seen. Left eye appeared normal. Optical coherence tomography (Spectralis; Heidelberg Engineering, Heidelberg, Germany) revealed presence of thick submacular hemorrhage in the right eye (Fig. I). Patient was diagnosed submacular hemorrhage in the right eye due to choroidal rupture following blunt trauma.

Three days after initial visit, pars plana vitrectomy was performed in the right eye under general anesthesia with subretinal injection of tissue plasminogen activator (rt-PA) and perfluoropropane tamponade. Briefly, after performing a 23-gauge micro-incision vitrectomy, $10 \mu \mathrm{g}$ rt-PA in $0.1 \mathrm{~mL}$ was injected subretinally using a 39-gauge subretinal infusion needle to liquefy the submacular hemorrhage. When injecting rt-PA, a small bubble of air was noticed under the retina (Fig. 2). Perfluorocarbon liquid was used to evacuate liquefied clot from the submacular space. Figure 3 shows displacement of the air bubble and liquefied clot from subfoveal area to the periphery. Before completing the operation, fluid-air exchange with com-

Address for correspondence: Zeynep Alkin, MD. Beyoglu Eye Training and Research Hospital, Bereketzade Cami Sok., 


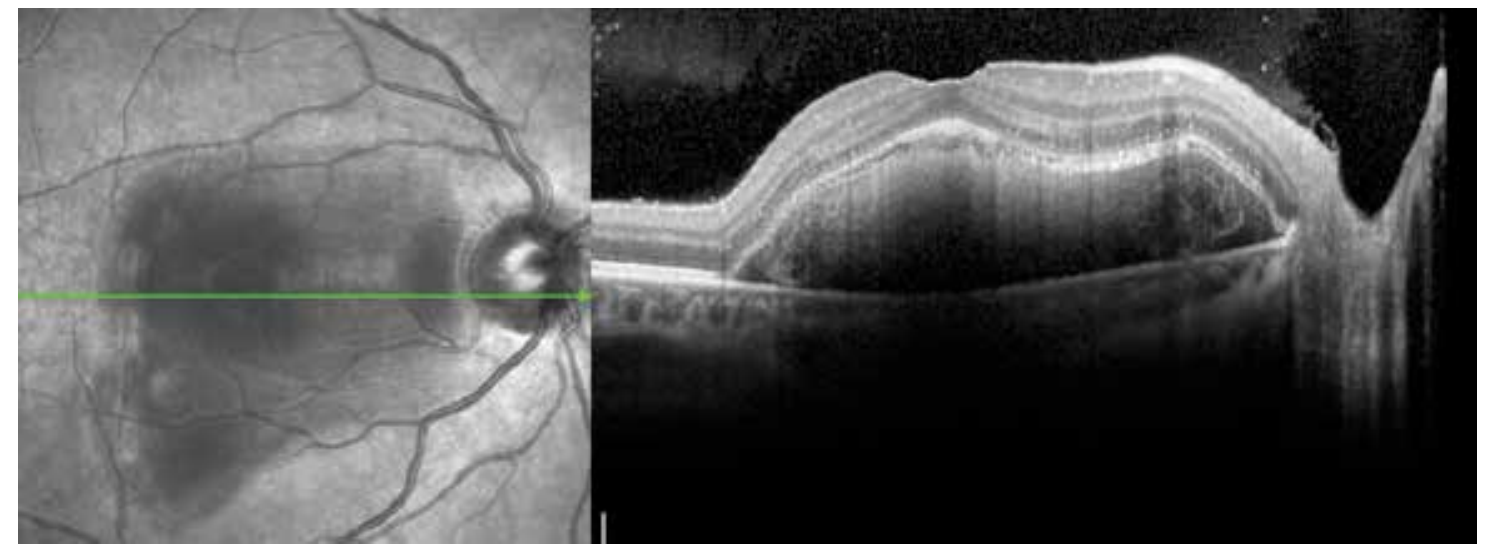

Figure I. Optical coherence tomography shows thick subretinal hemorrhage extending to the optic disc.

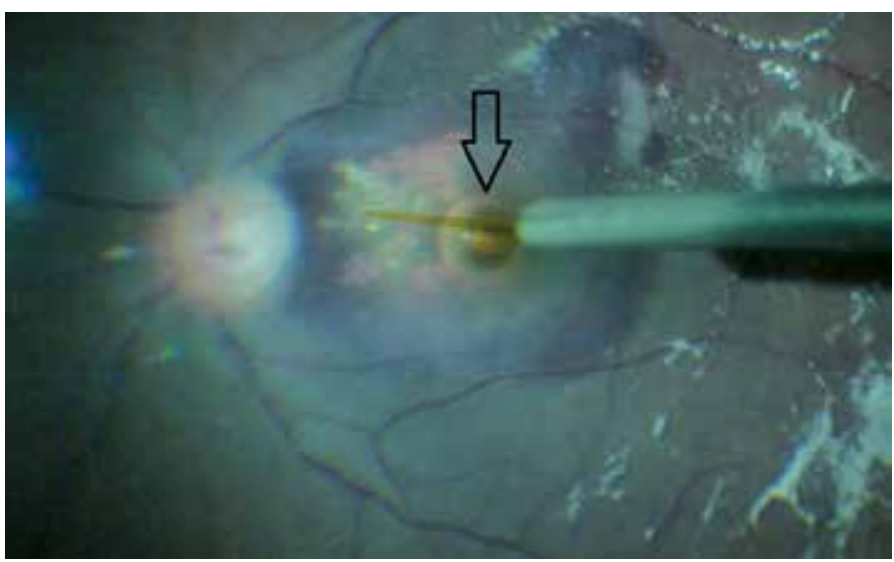

Figure 2. Fundus image shows small bubble of air under the retina (indicated by arrow).

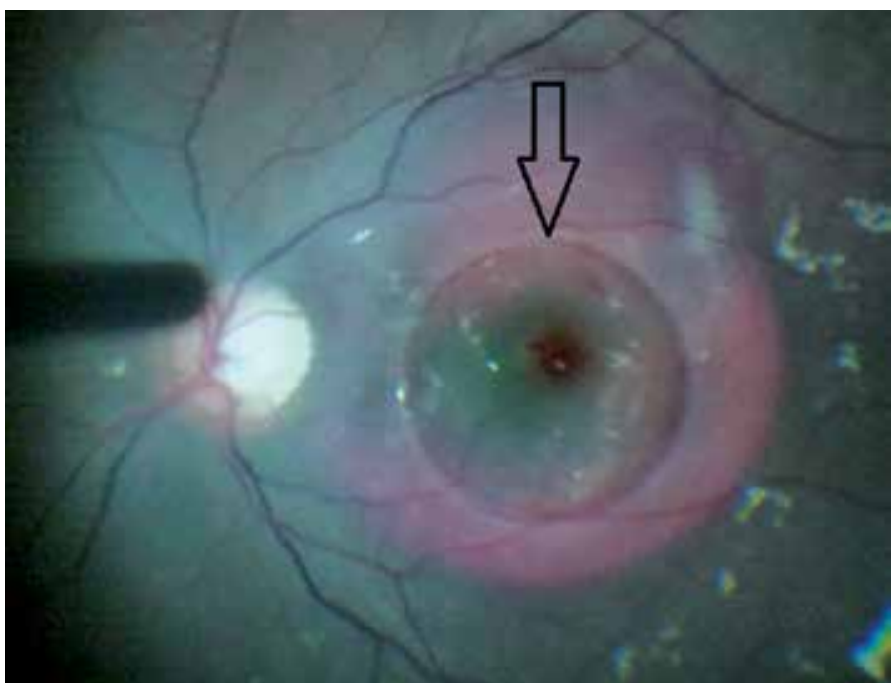

Figure 3. Fundus image shows displacement of the air bubble and liquefied clot from subfoveal area to the periphery.

plete removal of perfluorocarbon liquid was performed and nonexpansile concentration of $14 \%$ perfluoropropane was injected. Patient remained facedown for 3 days after surgery to displace the submacular hemorrhage. The day after the operation, most of the hemorrhage had moved to the inferior periphery.

One month after surgery, BCVA of right eye was 20/40. On fundus examination, thin remnants of the submacular hemorrhage and juxtafoveal choroidal rupture were seen. Unfortunately, OCT imaging revealed FTMH (Fig. 4). Three months after the first surgery, patient underwent second pars plana vitrectomy surgery with ILM peeling and intravitreal injection of perfluoropropane in the right eye under general anesthesia. One month after the second surgery, macular hole was closing and there was subretinal hyporeflective space, as can be seen in Figure 5 . Three months after the second surgery, BCVA in the right eye improved to 20/30. OCT imaging showed fully closed full-thickness macular hole and slight disintegrity in subfoveal ellipsoid zone (Fig. 6).

\section{Conclusion}

We successfully repaired FTMH in the eye of a 16-year-old boy with vitrectomy, ILM peeling, and perfluoropropane injection after surgery for traumatic submacular hemorrhage.

Treatment for submacular hemorrhage can be broadly divided into nonvitrectomizing and vitrectomizing techniques. Especially in young patients, wait-and-see approach or nonvitrectomizing techniques may be preferred for traumatic submacular hemorrhage. Pars plana vitrectomy is often combined with intravitreal injection of gas, or gas and rt-PA $(3,4)$. In this case, we chose vitrectomizing technique even though patient was 16-years-old because the submacular hemorrhage was very large and thick, and it was thought that nonvitrectomizing techniques would be insufficient. In early postoperative period, submacular hemorrhage moved out of the fovea and BCVA improved from 20/100 to 20/40. FTMH developed at that time. There are 2 typical mechanisms in pathogenesis of FTMH following trauma: direct foveal contusion or vitreous-mac- 


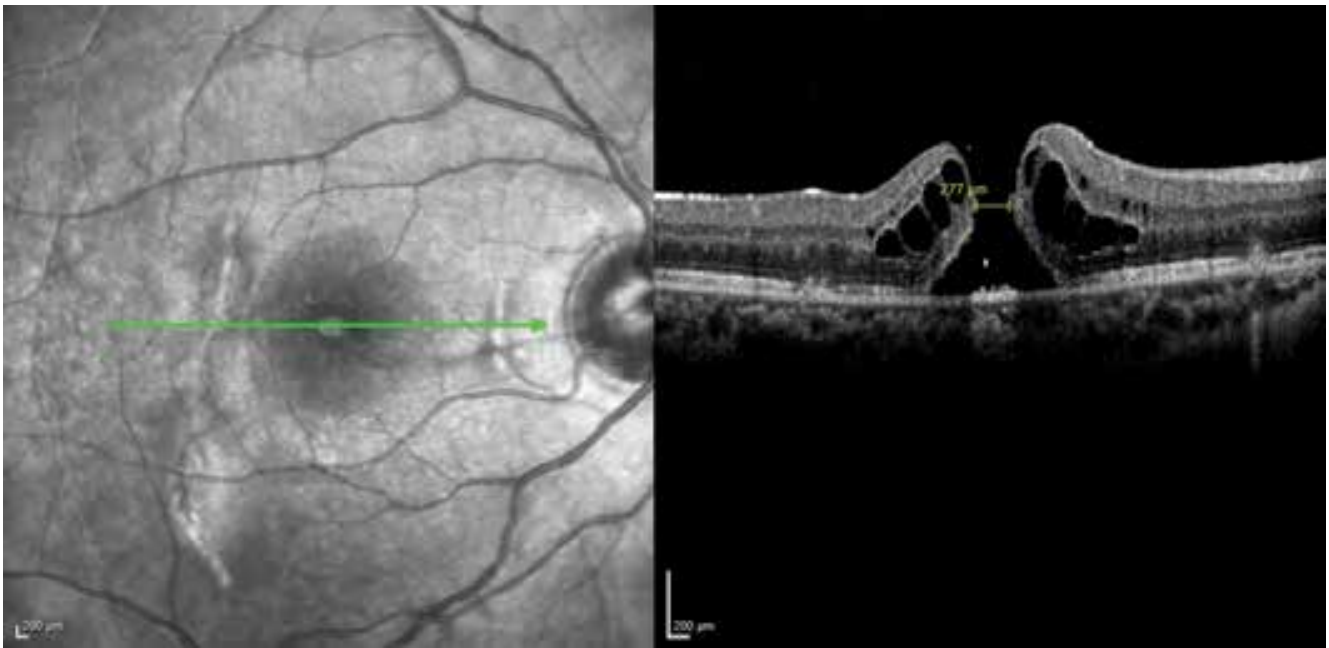

Figure 4. Optical coherence tomography shows full thickness macular hole curvilinear scar, temporal to the fovea, secondary to choroidal rupture I month after first surgery (minimum hole diameter is $277 \mu \mathrm{m}$ ).

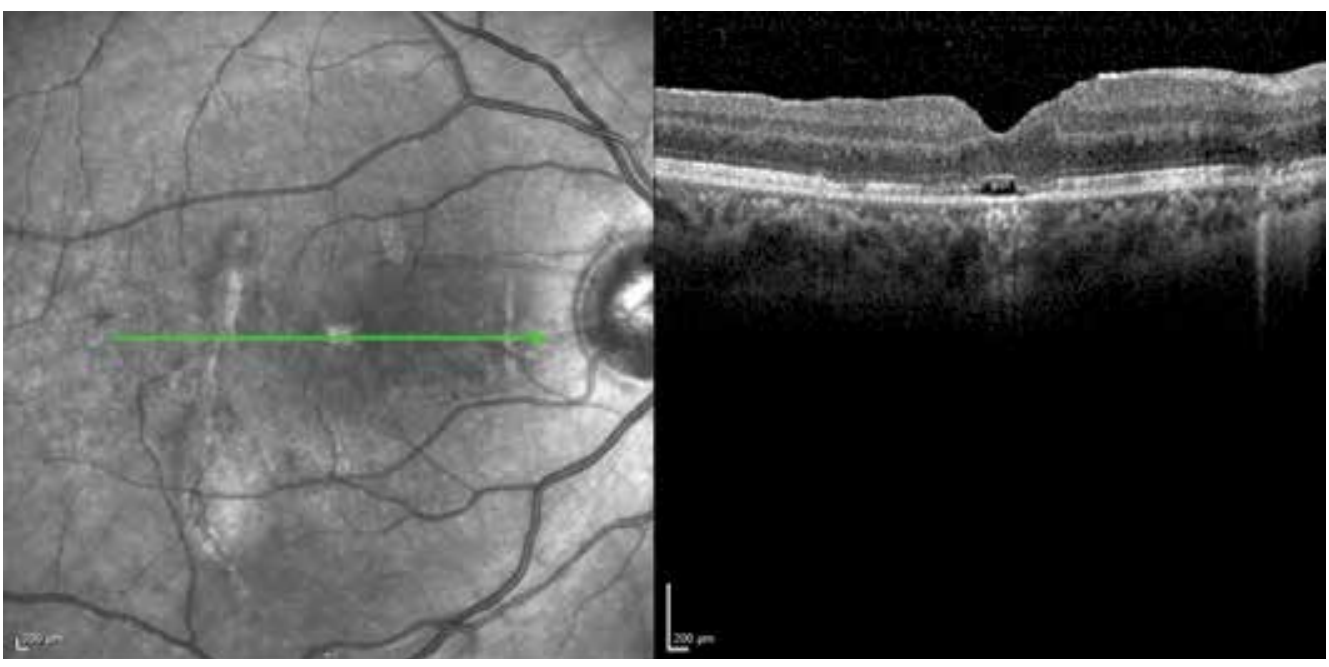

Figure 5. Optical coherence tomography shows closure of the macular hole with outer retinal subfoveal defect I month after second surgery.

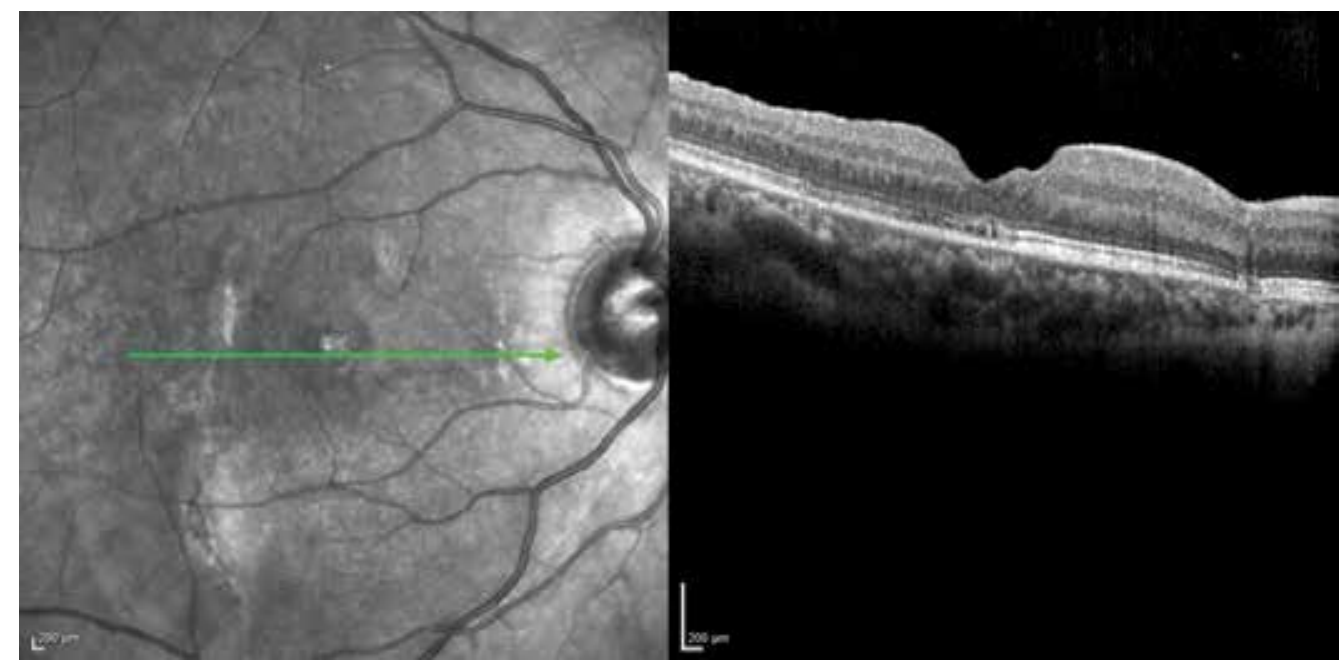

Figure 6. Optical coherence tomography shows total closure of the macular hole with disrupted outer ellipsoid zone. 
ular traction $(5,6)$. We speculate that in this case, however, FTMH may have been result of surface tension from small air bubble observed when sweeping retinal tissue with perfluorocarbon liquid during surgery.

Spontaneous closure occurs more easily in children, in cases of small macular holes $(100-200 \mu \mathrm{m})$, if the posterior hyaloid remains attached, or if fluid cuff is absent (710). However, 3 months after first surgery, FTMH was still present in this case. Therefore, we decided to perform second surgical intervention with ILM removal. Three months after second surgery, patient presented with macular hole closure and excellent visual acuity (20/30).

Rishi et al. reported on anatomical and functional outcomes following surgery in a case of traumatic macular hole (TMH) and subretinal hemorrhage. In their particular case, physical approximation of hole edges could have been facilitated by surgical removal of submacular hemorrhage and ILM removal as well. Seven months after surgery, they found closed TMH and improved BCVA from $20 / 125$ to $20 / 80$ (II).

In summary, pars plana vitrectomy with ILM peeling and gas tamponade seems to have been safe and effective choice of therapy of this unusual case of FTMH. Our interest in reporting this case is to highlight effectiveness and benefits of early interventional approach rather than observation.

\section{Disclosures}

Financial Disclosure: This case report was not supported by any company. None of the authors has financial or proprietary interests in any material or method mentioned. These data have not been previously published.

Peer-review: Externally peer-reviewed.

Conflict of Interest: None declared.

Authorship Contributions: Involved in design and conduct of the study (ZTA, AO, GE); preparation and review of the study (ZTA, AO, IP); data collection (ZTA, AO, AK).

\section{References}

I. Mitamura Y, Saito W, Ishida M, Yamamoto S, Takeuchi S. Spontaneous closure of traumatic macular hole. Retina 2001;21:385-9.

2. Yamashita T, Uemara A, Uchino E, Doi N, Ohba N. Spontaneous closure of traumatic macular hole. Am J Ophthalmol 2002; 133:230-5. Crossret

3. Holland D, Wiechens B. Intravitreal r-TPA and gas injection in traumatic submacular hemorrhage. Ophthalmologica 2004;2। 8:64-9. Crossre

4. Goldman DR, Vora RA, Reichel E. Traumatic choroidal rupture with submacular hemorrhage treated with pneumatic displacement. Retina 2014;34:1258-60. Crossre

5. Bonnin N, Cornut PL, Chaise F, Labeille E, Manificat HJ, Feldman $\mathrm{A}$, et al. Spontaneous closure of macular holes secondary to posterior uveitis: case series and a literature review. J Ophthalmic Inflamm Infect 2013;3:34. Crossre

6. Chen H, Zhang M, Huang S, Wu D. OCT and muti-focal ERG findings in spontaneous closure of bilateral traumatic macular holes. Doc Ophthalmol 2008; I 16:159-64. Crossre

7. Yamada H, Sakai A, Yamada E, Nishimura T, Matsumura M. Spontaneous closure of traumatic macular hole. Am J Ophthalmol 2002; 1 34:340-7. Crossre

8. Yeshurun I, Guerrero-Naranjo JL, Quiroz-Mercado H. Spontaneous closure of a large traumatic macular hole in a young patient. Am J Ophthalmol 2002;134:602-3. Crossre

9. Menchini U, Virgili G, Giacomelli G, Cappelli S, Giansanti F. Mechanism of spontaneous closure of traumatic macular hole: OCT study of one case. Retina 2003;23:104-6 Crossre

10. Carpineto P, Ciancaglini M, Aharrh-Gnama A, Agnifili L, Cerulli AM, Cirone D, et al. Optical coherence tomography and fundus microperimetry imaging of spontaneous closure of traumatic macular hole: a case report. Eur J Ophthalmol 2005; I5: I65-9.

II. Rishi P, Reddy S, Rishi E. Delayed, spontaneous conversion of type 2 closure to type I closure following surgery for traumatic macular hole associated with submacular hemorrhage. Oman J Ophthalmol 2012;5:189-90. Crossret 